\title{
Metal-on-metal hips and heart failure - can we relax?
}

With aging, we desire longevity - developments in public health, cancer and cardiovascular care support this. But we also desire improved quality of life with continued mobility and freedom from pain. Advances by the orthopaedic community over the years help us. One such advance in hip prostheses however proved disastrous. Conventional polyethylene/ceramic implant survival was deemed inadequate for fitter patients requiring greater longevity, so prostheses were developed with metal-on-metal (MoM) articular surfaces. This attractive concept was enthusiastically adopted, with more than 1.5 million implants globally, particularly in younger patients. Unfortunately, the consequences of even limited mechanical wear were unanticipated. Like a car engine with no oil, metal debris containing cobalt-chromium nanoparticles could be released into surrounding tissues causing pain, pseudotumours and premature failure. For clinicians and hospitals, this means substantial ongoing time/resource investment into annual surveillance and redo operations (early MoM failure rates are up to $15 \%$, with costs of over $£ 20$ million annually for revision surgery in the UK alone). For regulators and litigators, a minefield and field day respectively.

Alongside local complications, high plasma levels of cobalt and chromium nanoparticles led to concerns of systemic toxicity. Cobalt has long been known to cause cardiomyopathy in predisposed individuals. [1]Case reports have described heart failure in patients with MoM hips,[2] with some demonstrating cardiac recovery on implant revision implying causation. One small clinical study [3] led many to question whether these cases represented the tip of an iceberg. This was possible - MoM hip use and heart failure co-segregate in the elderly (UK prevalences in those over 75 years are $5 \%$ for hip replacement and $8 \%$ for heart failure); toxicity would be late after the implant (tissue metal levels need to accrue), there are no specific defining features, and heart failure symptoms may be masked by impaired mobility in a failing hip. Similarly, simple comparison of the heart failure incidence in MoM versus conventional implants would potentially be diluted because MoM recipients are typically younger and more active. Consequently regulators, in part driven by anxiety related to potential litigation, began to express major concern.

In this edition, Lodge and colleagues describe small increases in left ventricular volumes in MoM patients compared to controls, that relate to blood cobalt levels and renal function. Importantly however, echocardiographic and blood biomarkers of cardiac function were similar between patients and controls. However the relationship of blood cobalt levels and cardiac volumes with renal function warrants further exploration. Cobalt excretion appears not to be influenced by renal function, but renal deposition of cobalt may itself impair renal function. Subjects with even modest albuminuria have bigger hearts, so apparent cardiac remodeling here may primarily be mediated via renal abnormalities.

What should we as cardiologists take from this work? As with several other clinical/ largescale population studies, [4-6] this work failed to detect a strong signal for heart failure risk with MoM hips. Indeed one study from the largest joint registry (including data from greater than 500,000 patients) suggested lower heart failure risk in MoM patients. [5] One smaller epidemiological study found potentially higher risks of heart failure but only in a small subgroup of elderly men with one particular high risk prosthesis. [7] Currently, there is no evidence to advise routine cardiac screening of MoM patients and we think that 
patients should be reassured unless their blood levels are very high. Incidence even of rare cases in those with extreme plasma metal ion levels will also fall in coming years as increasingly vigilant screening recommendations means earlier revision surgery.[8] MoM bearings are now used in less than $1 \%$ of all hip replacements each year and have been since 2012.[9] However, albeit extremely rare, cobalt cardiomyopathy is one of few truly reversible causes of heart failure, and therefore in high risk patients should be considered.

But how does one go further and prove a negative to confidently allay all concerns? Prospective randomized clinical trials are not possible, given the known complications with MoM hips. Further observational cohort studies will help, but are confounded by both poor phenotyping of heart failure and the limitations of echocardiographic ejection fraction as the primary marker of heart failure.[10] Myocardial cobalt cannot be detected noninvasively (although T2* MRI may help), and the two biomarkers used routinely in cardiology lack specificity and have not been found to be useful in this context.[4] In subgroups at particular risk, these studies despite their limitations remain important. In the interim we should be reassured by the large scale epidemiological studies; but must remain alert for rare cases with high blood ion levels.

Are there wider lessons to learn for cardiology? Detection of cardiotoxicity in systemic disorders remains suboptimal. Modern medicine embraces siloed healthcare delivered by distinct specialities, with limited communication hindering clinical care and research. Lodge et al should be congratulated on this cross-disciplinary work demanding close cardiology and orthopaedic collaboration. Cardiology faces a similar challenge in cancer patients. The new expanding sub-speciality of cardio-oncology enables earlier detection and treatment of cardiac complications related to cancer therapy, and generally means that higher risk patients receive cancer treatments that would otherwise be denied. With the explosion of novel cancer treatments emerging including immunotherapies,[10] robust inter-disciplinary interaction and adaptive care and research is essential - so it may be with 'cardioorthopaedics'.

There are wider lessons. One positive to be drawn from this episode is the value of comprehensive device registries such as the National Joint Registry of England and Wales, which now includes data from $95 \%$ of new hip implants. Although device registries exist within cardiology, linkage to outcomes is not always routine, and the pace of device innovation often exceeds registry updates. The advent of machine learning in big data analysis means such registries offer even greater potential for early detection of safety signals in the near future.

In summary, despite high early failure rates with MoM implants, significant cardiac dysfunction is uncommon and patients should be re-assured. Within cardiology however we need better non-invasive diagnosis and stratification of heart failure, both to aid earlier detection of rare causes such as cobalt cardiomyopathy, and to develop targeted therapies. Finally, we must remember that many of the devices that we implant into our patients are there for life, and initial pre-marketing safety tests in selected populations may miss the unpredictable late complications. There is no room for complacency. 
[1] C.S. Alexander, Cobalt-beer cardiomyopathy. A clinical and pathologic study of twenty-eight cases., Am. J. Med. 53 (1972) 395-417. http://www.ncbi.nlm.nih.gov/pubmed/4263183 (accessed June 27, 2018).

[2] S.M. Bradberry, J.M. Wilkinson, R.E. Ferner, Systemic toxicity related to metal hip prostheses, Clin. Toxicol. 52 (2014) 837-847. doi:10.3109/15563650.2014.944977.

[3] J.R. Prentice, M.J. Clark, N. Hoggard, A.C. Morton, C. Tooth, M.N. Paley, I. Stockley, M. Hadjivassiliou, J.M. Wilkinson, Metal-on-Metal Hip Prostheses and Systemic Health: A Cross-Sectional Association Study 8 Years after Implantation, PLoS One. 8 (2013) e66186. doi:10.1371/journal.pone.0066186.

[4] R. Berber, A. Abdel-Gadir, S. Rosmini, G. Captur, S. Nordin, V. Culotta, L. Palla, P. Kellman, G.W. Lloyd, J.A. Skinner, J.C. Moon, C. Manisty, A.J. Hart, Assessing for Cardiotoxicity from Metal-on-Metal Hip Implants with Advanced Multimodality Imaging Techniques, J. Bone Joint Surg. Am. 99 (2017). doi:10.2106/JBJS.16.00743.

[5] S.A. Sabah, J.C. Moon, S. Jenkins-Jones, C.L. Morgan, C.J. Currie, J.M. Wilkinson, M. Porter, G. Captur, J. Henckel, N. Chaturvedi, P. Kay, J.A. Skinner, A.H. Hart, C. Manisty, The risk of cardiac failure following metalon-metal hip arthroplasty, Bone Jt. J. 100B (2018). doi:10.1302/0301-620X.100B1.BJJ-2017-1065.R1.

[6] L.H. Goodnough, A. Bala, J. Huddleston, S.B. Goodman, W.J. Maloney, D.F. Amanatullah, Metal-on-metal total hip arthroplasty is not associated with cardiac disease., Bone Joint J. 100-B (2018) 28-32. doi:10.1302/0301-620X.100B1.BJJ-20170366.R1.

[7] M.H. Gillam, N.L. Pratt, M.C.S. Inacio, E.E. Roughead, S. Shakib, S.J. Nicholls, S.E. Graves, Heart failure after conventional metal-on-metal hip replacements., Acta Orthop. 88 (2017) 2-9. doi:10.1080/17453674.2016.1246276.

[8] MHRA, All metal-on-metal (MoM) hip replacements: updated advice for follow-up of patients - (MDA/2017/018), Med. Healthc. Prod. Regul. Agency. (2017). https://www.gov.uk/drug-device-alerts/all-metal-on-metal-mom-hip-replacementsupdated-advice-for-follow-up-of-patients.

[9] N.I. National Joint Registry (NJR) for England, Wales, National Joint Registry (NJR) for England, Wales, Northern Ireland and the Isle of Man 13th Annual Report. 2017, (2017). http://www.njrreports.org.uk/Portals/0/PDFdownloads/NJR 14th Annual Report 2017.pdf.

[10] Moslehi, Cardiovascular Toxic Effects of Targeted Cancer Therapies, NEJM. 15 (2016). doi:10.1056/NEJMra1100265. 